ISSN 0258-7122 (Print), 2408-8293 (Online)

Bangladesh J. Agril. Res. 42(3): 425-436, September 2017

\title{
COMBINING ABILITY ESTIMATES IN MAIZE (ZEA MAYS L.) THROUGH LINE $\times$ TESTER ANALYSIS
}

\author{
S. AHMED ${ }^{1}$, S. BEGUM ${ }^{2}$, M. A. ISLAM ${ }^{3}$ \\ M. RATNA ${ }^{4}$ AND M. R. KARIM ${ }^{5}$
}

\begin{abstract}
Eighteen advanced $\mathrm{S}_{4}$ lines of maize extracted from NK46 were evaluated through line $\times$ tester method by using two testers for grain yield and its components. General combining ability (GCA) and specific combining ability (SCA) effects of crosses were determined to evaluate the prospective inbred lines. Highly significant genotypic differences for all of six characters, i.e. days to $50 \%$ tasselling, Days to $50 \%$ silking, plant height, ear height, 1000 kernel weight, and yield indicated presence of wide range of variability among the genotypes for those traits. Non-additive gene action was predominant. Four lines for days to $50 \%$ tasselling, one for days to $50 \%$ silking, two for plant height, one for ear height, three for 1000 kernel weight, and four for yield were found with significant GCA effects in desired direction. For days to tasselling, days to silking, plant height and ear height, none of 36 cross combinations confirmed significant SCA effects. Five combinations showed significant positive SCA effects for 1000 kernel weight. For yield two crosses showed significant positive SCA. Considering the results of present study, based on GCA and SCA analysis of observed characters seven lines namely, NK46-2, NK46-4, NK46-10, NK4613, NK46-18, NK46-43 and NK46-44 were selected for further breeding program
\end{abstract}

Keywords: Zea mays L., Line $\times$ Tester, combining ability.

\section{Introduction}

Maize (Zea mays. L) is the third most important cereal crop with abundant natural diversity. In Bangladesh, its area and production is increasing. Maize is a highly allogamous crop and it has been successfully exploited for the production of hybrids. Superior inbred lines with good combining ability are prerequisite for development of superior hybrids. Maize breeding methodology generally involves the development of inbred lines from heterogeneous source population through continuous selfing. Selection of segregants at early generations is very important to reduce the number of inbred lines to a manageable size. Combining ability at early and advanced generation in maize is an excellent tool which helps

${ }^{1}$ Scientific Officer, Biotechnology Division, Bangladesh Agricultural Research Institute (BARI), Gazipur-1701, ${ }^{2}$ Scientific Officer, Plant Breeding Division, BARI, Gazipur1701, ${ }^{3}$ Senior Scientific Officer, Plant Breeding Division, Regional Agricultural Research Station, BARI, Jamalpur-2000, ${ }^{4}$ Scientific Officer, Spices Research Sub-Centre, BARI, Faridpur-7800, ${ }^{5}$ Scientific Officer, Horticulture Research Centre, BARI, Gazipur-1701, Bangladesh. 
to discern the goal and direction in a breeding program (Manonmani and Khan, 2003). Its role is important to decide parents, crosses and appropriate breeding procedure to be followed to select desirable segregants (Salgotra et al., 2009). It has been reported that selection during inbreeding based on the performance of test cross progeny is highly effective in improving the GCA of inbred lines. In this context, LxT analysis (Kempthrone, 1957) has widely been used for evaluation of large number of lines by crossing them with testers to obtain superior inbred lines with desired traits. The present study involving a line $x$ tester analysis was aimed to determine the general combining ability (GCA) and specific combining ability (SCA) of developed crosses for different traits and to explore the superior lines with earliness, low plant and ear height with higher yield for advancing to the next generation.

\section{Materials and Method}

Eighteen lines of maize were selected from $\mathrm{S}_{4}$ lines which were developed from a source population of NK46 through recycling (repeated inbreeding) for four generations. Two inbred lines with diverse genetic base namely BIL28 (Jahan et al. 2014) and BIL79 were selected as testers. During rabi, 2012-13 the hybridization was performed following Line $\times$ Tester mating design using $18 \mathrm{~S}_{4}$ lines (listed in Table 1) as female and testers as male parents to obtain 36 cross combinations. The 36 cross combinations and 18 advanced lines and two testers were grown in an Alpha lattice design with two replications with spacing of $75 \mathrm{x}$ $20 \mathrm{~cm}$ at Regional Agricultural Research Station, Bangladesh Agricultural Research Institute, Hathazari, Chittagong, Bangladesh during rabi, 2013-14. Seeds were sown in 29 November, 2013. One border row was used at each end of the replication to minimize the border effect. Fertilizers were applied @ 250, $120,120,40,5$ and $1 \mathrm{~kg} / \mathrm{ha}$ of $\mathrm{N}, \mathrm{P}_{2} \mathrm{O}_{5}, \mathrm{~K}_{2} \mathrm{O}, \mathrm{S}, \mathrm{Zn}$ and $\mathrm{B}$ respectively. One third urea and total amount of other fertilizers were applied at final land preparation. The remaining urea was applied in two installments as top dressing. Standard agronomic practices including two weeding and four irrigations and plant protection measures were followed to raise a healthy crop. The data were recorded on 10 randomly selected plants for quantitative characters viz. days to $50 \%$ tasselling, days to 50\% silking, plant height $(\mathrm{cm})$, ear height $(\mathrm{cm}), 1000$ grain weight $(\mathrm{g})$, and grain yield $(\mathrm{g})$. Combining ability analysis was done as per the method given by Kempthrone (1957).

\section{Results and Discussion}

The analysis of variance presented in Table 2 revealed highly significant differences among the genotypes for all the characters, suggesting sufficient genetic variability among the genotypes. Highly significant differences between parents (lines and testers) and interactions of parents and crosses for all the traits indicated wide range of variability present among them. Variability between crosses is highly significant for days to tasselling, 1000 kernel weight and yield 
which were in conformity with Shushay et al. (2013). Lines differed at highly significant level in favor of days to tasselling, days to silking, ear height, and yield and at significant level for plant height which represented prevalence of substantial variability in them. Testers differed significantly for days to $50 \%$ tasselling and days to $50 \%$ silking where difference between LinexTester was highly significant for 1000 kernel weight and yield (Table 2). Jahan et al. (2014) also found significant difference for days to tasselling, and silking between testers and for yield between LinexTester interaction. Similar genotypic difference for grain weight, grain yield and other characters were reported by Narro et al. (2003) and Sofi and Rathor (2006).

Table 1. List of 18 selected $S_{4}$ lines and two testers used in LinexTester mating

\begin{tabular}{|c|c|c|c|}
\hline S1. No. & Parents & S1. No. & Parents \\
\hline \multicolumn{3}{|c|}{ Line } & Line \\
\hline 1 & NK46-2 & 10 & NK46-18 \\
\hline 2 & NK46-4 & 11 & NK46-21 \\
\hline 3 & NK46-6 & 12 & NK46-22 \\
\hline 4 & NK46-7 & 13 & NK46-23 \\
\hline 5 & NK46-8 & 14 & NK46-34 \\
\hline 6 & NK46-10 & 15 & NK46-39 \\
\hline 7 & NK46-12 & 16 & NK46-40 \\
\hline 8 & NK46-13 & 17 & NK46-43 \\
\hline 9 & NK46-14 & 18 & NK46-44 \\
\hline \multicolumn{4}{|c|}{ Tester } \\
\hline 1 & \multicolumn{3}{|c|}{ BIL 28} \\
\hline 2 & \multicolumn{3}{|c|}{ BIL 79} \\
\hline
\end{tabular}

The higher estimation of dominance variance $\left(\sigma^{2} \mathrm{sca}\right)$ as compared to additive variance $\left(\sigma^{2} \mathrm{gca}\right)$ which produced the below one ratio of $\sigma^{2} \mathrm{gca}$ to $\sigma^{2} \mathrm{sca}$ for all the six characters (Table 2) probably due to predominance of non-additive gene action suggested the scope of improvement of these characters through heterosis breeding. Similar non-additive gene action for all traits under this study was also reported by Talukder et al. (2016). Suneetha et al. (2000) got non-additive gene action for days to 50\% tasselling and days to 50\% silking. Amin et al. (2014a) found non-additive gene action for plant height, 1000 grain weight and yield. Singh and Singh (1998) reported non-additive gene action for plant height, ear height, 1000 grain weight where Mahto and Ganguly (2001) also reported non additive gene action for grain yield that supported the present study. However, Amin et al. (2014b) found similar results for days to silking, and yield but different results i.e. additive gene action for plant height, ear height and grain weight which might be due to use of different genotypes and mating design in their study. 
Table 2. ANOVA showing mean squares and estimates of variance for grain yield and other characters in maize

\begin{tabular}{|c|c|c|c|c|c|c|c|}
\hline Source & $\mathrm{df}$ & $\begin{array}{c}\text { Days to } \\
50 \% \\
\text { tasselling }\end{array}$ & $\begin{array}{c}\text { Days to } \\
50 \% \\
\text { silking }\end{array}$ & $\begin{array}{c}\text { Plant } \\
\text { height }(\mathrm{cm})\end{array}$ & $\begin{array}{l}\text { Ear height } \\
\quad(\mathrm{cm})\end{array}$ & $\begin{array}{c}1000- \\
\text { kernel } \\
\text { weight }(\mathrm{g})\end{array}$ & $\begin{array}{l}\text { Yield } \\
(\mathrm{t} / \mathrm{ha})\end{array}$ \\
\hline Genotypes & 55 & $36.83 * *$ & $32.83 * *$ & $901.53 * *$ & $553.74 * *$ & $2623.53 * *$ & $8.86 * *$ \\
\hline Parents (P) & 19 & $27.75 * *$ & $26.87 * *$ & $1142.34 * *$ & $716.35^{* *}$ & $1943.51 * *$ & $4.33 * *$ \\
\hline $\mathrm{P}$ vs $\mathrm{C}$ & 1 & $860.89 * *$ & $857.59 * *$ & $18003.01 * *$ & $10520.76 * *$ & $10142.42 * *$ & $190.96 * *$ \\
\hline $\begin{array}{l}\text { Crosses } \\
\text { (C) }\end{array}$ & 35 & $18.22 * *$ & 12.50 & 282.19 & 180.70 & $2777.86 * *$ & $6.12 * *$ \\
\hline Lines (L) & 17 & $29.24 * *$ & $20.89 * *$ & $401.45 *$ & $315.16^{* *}$ & 3190.79 & $8.29 *$ \\
\hline Testers (T) & 1 & $29.39 *$ & $17.01 *$ & 5.31 & 13.52 & 5341.12 & 1.01 \\
\hline $\mathrm{L} \times \mathrm{T}$ & 17 & 6.54 & 3.84 & 179.23 & 56.07 & $2214.15 * *$ & $4.25 * *$ \\
\hline Error & 55 & 5.92 & 8.06 & 183.32 & 159.99 & 330.79 & 1.47 \\
\hline \multicolumn{8}{|c|}{ Estimates of component of variances } \\
\hline$\sigma^{2} \mathrm{~g}$ (Line) & - & 5.68 & 4.26 & 55.55 & 64.77 & 244.16 & 1.01 \\
\hline$\sigma^{2} \mathrm{~g}($ Tester $)$ & - & 0.63 & 0.37 & 4.83 & 1.18 & 86.86 & 0.09 \\
\hline$\sigma^{2} \mathrm{gca}$ & - & 0.22 & 0.16 & 1.93 & 2.33 & 10.54 & 0.03 \\
\hline$\sigma^{2} \mathrm{sca}$ & - & 0.31 & 2.11 & 2.05 & 51.96 & 941.68 & 1.39 \\
\hline $\begin{array}{c}\sigma^{2} \mathrm{gca} / \\
\sigma^{2} \mathrm{sca}\end{array}$ & - & 0.71 & 0.08 & 0.94 & 0.04 & 0.01 & 0.03 \\
\hline
\end{tabular}

$* \mathrm{P}=0.05$ and $* * \mathrm{P}=0.01$

The contribution of lines, testers and their interactions to total variances were presented in Table 3. The proportional contribution of lines and interactions to total variances were much higher than testers in all the traits. However, the contributions of lines were higher than the interactions to total variances for all the characters. This suggested female parents contributed maximum to total variance in maize, followed by interaction. Testers contributed the lowest to total variance, which is in agreement with Amiruzzaman and Amin (2011a, b), Parvin (2009), Rissi et al. (1991) and Talukder and Banik (2012).

Table 3. Proportional contribution of lines, testers and their interactions to total variance in maize

\begin{tabular}{c|c|c|c|c|c|c}
\hline Source & $\begin{array}{c}\text { Days to } \\
\text { tasselling }\end{array}$ & $\begin{array}{c}\text { Days to } \\
\text { silking }\end{array}$ & $\begin{array}{c}\text { Plant height } \\
(\mathrm{cm})\end{array}$ & $\begin{array}{c}\text { Ear height } \\
(\mathrm{cm})\end{array}$ & $\begin{array}{c}\text { 1000-kernel } \\
\text { weight }(\mathrm{g})\end{array}$ & $\begin{array}{c}\text { Yield } \\
(\mathrm{t} / \mathrm{ha})\end{array}$ \\
\hline Line & 77.96 & 81.19 & 69.10 & 84.71 & 55.79 & 65.79 \\
Tester & 4.61 & 3.89 & 0.05 & 0.21 & 5.49 & 0.47 \\
Line $\times$ Tester & 17.43 & 14.92 & 30.85 & 15.07 & 38.71 & 33.74 \\
\hline
\end{tabular}




\section{General combining ability effects}

Selection of parents with good general combining ability is a prime requisite for any successful breeding program especially for heterosis breeding. The general combining ability effects and per se performance of parents (line and tester) were presented in Table 4. Negative GCA effects are desired for days to tasselling, days to silking, plant height and ear height; but in case of yield the choice for GCA effects is in positive direction. The line NK46-10 and NK46-40 exhibited highly significant negative GCA effects for days to tasselling where NK46-18 and NK46-44 showed significant negative GCA effects. For days to silking only NK46-40 showed highly significant negative GCA effects. These lines can be utilized for evolving earliness. Hussain et al. (2003), Roy et al. (1998), and Uddin et al. (2006) also observed similar phenomenon in their study. For plant height, line NK46-22 contributed significant negative GCA effects where NK464 was with significant negative GCA effect for both plant height and ear height. This indicated that these parents can be utilized for developing dwarf hybrids to reduce yield loss due to root and stem lodging. One line (NK46-39) with highly significant positive GCA effects and two lines (NK46-8 and NK46-23) with significant positive GCA in favor of 1000 kernel weight found in the study may be important for developing bold grain, whereas other five lines showed significant negative GCA for this trait. Similar to the current findings both positive and negative GCA effects for 1000 kernel weight were reported by Koppad (2007) and Wali et al. (2010). Parents with good GCA effects for 1000kernel weight were not found with good GCA for yield. It might occur due to the effects of other yield contributing characters like ear/plant, length of ear, row/ear, kernel/row etc. Highly significant positive GCA effects for yield in NK46-2, NK46-13, NK46-18, NK46-43 and NK46-44 indicated that these parents were good general combiner. These lines were promising to be used for exploiting more positive alleles for yield. Significant GCA effect for yield in maize was also reported by Ivy and Hawlader (2000) and Paul and Duara (1991). The lines with high GCA effects representing additive gene action in inheritance of traits, may be utilized in hybridization program to improve respective traits through transgressive segregation.

\section{Specific combining ability effects (SCA)}

Specific combining ability and mean of the crosses for grain yield, its components and other characters were presented in Table 5. In this investigation, none of the crosses exhibited significant negative or positive SCA effects for days to 50\% tasselling, 50\% silking, plant height and ear height, although some crosses involved significant negative general combining parents. In case of maize, negative value is expected for these traits to develop early and short statured plant. Sixteen crosses revealed negative but non-significant SCA effects for both days to tasselling and silking. Nine crosses were non-significant negative specific combiner for both plant height and ear height. Uddin et al. (2006) also 
reported failure of some crosses to show significant negative SCA effects for plant height and ear height while parents were found with significant negative GCA effects. Significant positive SCA effects were found in NK46-8 $\times$ BIL28, NK46-13×BIL79, NK46-22×BIL79, NK46-40×BIL79 and NK46-43×BIL28, for 1000-kernel weight where only the parent NK46-8 was with good GCA effects for this trait. Although 1000-kernel weight is one of the important yield contributing characters the crosses with high SCA effects for this trait failed to give high SCA effects for yield. It might be due to the effects of other yield contributing characters. Positive SCA effect is expected for yield and NK46$4 \times$ BIL79 and NK46-10×BIL28 combinations exhibited significant positive SCA effects which were in desired direction. But the parents confirming significant positive GCA effects could not produce cross combinations with significant positive SCA. Das and Islam (1994), Shushay et al. (2013) and Uddin et al. (2006) found crosses with significant positive SCA effects for yield. The present results were in line with the earlier findings of Ivy and Howlader (2000) where they stated that good general combiner do not always show high SCA effects in their crosses. On the contrary Paul and Duara (1991) reported that parents with high GCA always produce hybrids with high SCA.

Table 4. General combining ability (GCA) effects and mean of parents for grain yield, yield components and other characters in maize

\begin{tabular}{c|c|c|c|c|c|cc}
\hline \multirow{2}{*}{$\begin{array}{c}\text { Sl. } \\
\text { No. }\end{array}$} & \multirow{2}{*}{ Parents } & \multicolumn{2}{c|}{$\begin{array}{c}\text { Days to 50\% } \\
\text { tasselling }\end{array}$} & \multicolumn{2}{c|}{ Days to 50\% silking } & \multicolumn{2}{c}{ Plant height (cm) } \\
\cline { 3 - 8 } & & GCA & mean & GCA & mean & GCA & mean \\
\hline \multicolumn{7}{c}{ Tester } \\
\hline 1 & BIL 28 & 0.64 & 101.00 & 0.49 & 105.50 & -0.27 & 191.20 \\
2 & BIL 79 & -0.64 & 94.50 & -0.49 & 98.50 & 0.27 & 195.10 \\
& SE $_{\left(g_{i}\right)}$ & 0.41 & - & 0.47 & - & 2.26 & - \\
& SE $_{\left(g_{i}-g_{j}\right)}$ & 0.57 & - & 0.67 & - & 3.19 & - \\
\hline \multicolumn{7}{c}{ Line } \\
\hline 1 & NK46-2 & -0.89 & 95.00 & -1.13 & 98.50 & -3.67 & 159.75 \\
2 & NK46-4 & $2.86^{*}$ & 107.50 & 2.88 & 110.50 & $-16.96^{*}$ & 130.00 \\
3 & NK46-6 & 0.36 & 100.50 & 0.63 & 104.50 & -9.97 & 171.00 \\
4 & NK46-7 & -0.89 & 103.00 & 0.13 & 107.00 & -5.72 & 164.70 \\
5 & NK46-8 & -1.89 & 97.50 & -0.88 & 101.00 & -7.27 & 206.70 \\
6 & NK46-10 & $-3.64^{* *}$ & 102.00 & -2.63 & 106.50 & 0.63 & 146.10 \\
7 & NK46-12 & $3.11^{*}$ & 102.50 & $3.13^{*}$ & 105.50 & -1.02 & 174.90 \\
8 & NK46-13 & $4.61 * *$ & 105.00 & 2.88 & 107.50 & 0.38 & 148.45 \\
9 & NK46-14 & -0.64 & 96.00 & -1.63 & 99.50 & $18.45^{*}$ & 200.70 \\
10 & NK46-18 & $-2.89^{*}$ & 99.00 & -2.13 & 102.50 & 14.13 & 191.60 \\
\hline
\end{tabular}




\begin{tabular}{|c|c|c|c|c|c|c|c|}
\hline \multirow{2}{*}{$\begin{array}{l}\text { Sl. } \\
\text { No. }\end{array}$} & \multirow{2}{*}{ Parents } & \multicolumn{2}{|c|}{$\begin{array}{c}\text { Days to } 50 \% \\
\text { tasselling }\end{array}$} & \multicolumn{2}{|c|}{ Days to $50 \%$ silking } & \multicolumn{2}{|c|}{ Plant height $(\mathrm{cm})$} \\
\hline & & GCA & mean & GCA & mean & GCA & mean \\
\hline 11 & NK46-21 & $2.86^{*}$ & 97.50 & 1.88 & 100.50 & 15.83 & 171.60 \\
\hline 12 & NK46-22 & 3.11 & 97.50 & 2.88 & 101.00 & $-2.12 *$ & 163.50 \\
\hline 13 & NK46-23 & -0.89 & 99.00 & -0.88 & 103.00 & -2.67 & 177.10 \\
\hline 14 & NK46-34 & 1.11 & 100.50 & 0.63 & 103.00 & -6.59 & 158.04 \\
\hline 15 & NK46-39 & -1.64 & 92.50 & -1.88 & 96.50 & -0.66 & 209.75 \\
\hline 16 & NK46-40 & $-4.64 * *$ & 96.00 & $-4.13^{* *}$ & 98.50 & 7.73 & 182.50 \\
\hline 17 & NK46-43 & $2.61 *$ & 100.00 & 2.63 & 103.00 & -12.72 & 212.10 \\
\hline 18 & NK46-44 & $-2.64 *$ & 102.00 & -2.38 & 105.50 & 12.28 & 213.50 \\
\hline & $\mathrm{SE}_{\left(g_{i}\right.}$ & 1.22 & - & 1.42 & - & 6.77 & - \\
\hline & $\mathrm{SE}_{\left(g_{i-} g_{j}\right)}$ & 1.72 & - & 2.01 & - & 9.57 & - \\
\hline
\end{tabular}

$* \mathrm{P}=0.05$ and $* * \mathrm{P}=0.01$

Table 4. cont'd.

\begin{tabular}{|c|c|c|c|c|c|c|c|}
\hline \multirow{2}{*}{$\begin{array}{l}\text { Sl. } \\
\text { No. }\end{array}$} & \multirow{2}{*}{ Parents } & \multicolumn{2}{|c|}{ Ear height $(\mathrm{cm})$} & \multicolumn{2}{|c|}{$\begin{array}{l}1000 \text { kernel weight } \\
(\mathrm{g})\end{array}$} & \multicolumn{2}{|c|}{ Yield (t/ha) } \\
\hline & & GCA & mean & GCA & mean & GCA & mean \\
\hline \multicolumn{8}{|c|}{ Tester } \\
\hline 1 & BIL 28 & 0.43 & 94.40 & 8.61 & 297.50 & -0.12 & 7.33 \\
\hline 2 & BIL 79 & -0.43 & 93.50 & -8.61 & 255.50 & 0.12 & 4.86 \\
\hline & $\mathrm{SE}_{\left(g_{i}\right)}$ & 2.11 & - & 3.03 & - & 0.20 & - \\
\hline & $\mathrm{SE}_{\left(g_{i-} g_{j)}\right.}$ & 2.98 & - & 4.29 & - & 0.29 & - \\
\hline \multicolumn{8}{|c|}{ Line } \\
\hline 1 & NK46-2 & -12.12 & 61.63 & 10.67 & 367.50 & $1.66^{* *}$ & 7.25 \\
\hline 2 & NK46-4 & $-16.71 *$ & 60.25 & $-28.05 * *$ & 311.60 & -0.74 & 6.62 \\
\hline 3 & NK46-6 & -2.87 & 76.70 & $-24.80 *$ & 268.00 & $-2.55 * *$ & 5.96 \\
\hline 4 & NK46-7 & -1.46 & 77.80 & -17.30 & 250.50 & 0.34 & 4.38 \\
\hline 5 & NK46-8 & -5.76 & 107.00 & $58.43^{*}$ & 279.00 & 0.48 & 7.97 \\
\hline 6 & NK46-10 & -6.26 & 59.30 & 4.56 & 331.00 & -0.35 & 6.45 \\
\hline 7 & NK46-12 & -1.96 & 75.70 & 3.13 & 298.00 & 0.36 & 5.64 \\
\hline 8 & NK46-13 & -2.61 & 52.00 & 11.50 & 317.50 & $1.92 * *$ & 6.77 \\
\hline 9 & NK46-14 & 12.24 & 84.70 & $-46.37 * *$ & 322.31 & $-1.64 *$ & 9.97 \\
\hline 10 & NK46-18 & $15.54 *$ & 90.70 & 12.95 & 338.54 & $2.21 * *$ & 6.28 \\
\hline 11 & NK46-21 & 10.89 & 80.50 & 18.20 & 322.94 & 0.79 & 8.92 \\
\hline 12 & NK46-22 & -6.96 & 61.30 & $-34.05^{* *}$ & 352.50 & $-2.10 * *$ & 6.83 \\
\hline
\end{tabular}




\begin{tabular}{c|c|c|c|c|c|c|c}
\hline \multirow{2}{*}{$\begin{array}{c}\text { Sl. } \\
\text { No. }\end{array}$} & \multirow{2}{*}{ Parents } & \multicolumn{2}{|c|}{ Ear height (cm) } & \multicolumn{1}{c|}{$\begin{array}{r}1000 \text { kernel weight } \\
(\mathrm{g})\end{array}$} & \multicolumn{2}{c}{ Yield (t/ha) } \\
\cline { 3 - 8 } & & GCA & mean & GCA & mean & GCA & mean \\
\hline 13 & NK46-23 & -1.61 & 70.90 & $24.95^{*}$ & 331.50 & -0.62 & 5.76 \\
14 & NK46-34 & 7.34 & 60.88 & -9.53 & 298.50 & -1.21 & 5.16 \\
15 & NK46-39 & -2.61 & 110.40 & $46.62^{* *}$ & 339.50 & -0.02 & 7.06 \\
16 & NK46-40 & 4.89 & 72.60 & $-37.74 * *$ & 297.00 & -1.44 & 8.74 \\
17 & NK46-43 & -2.86 & 114.10 & 6.95 & 293.00 & $1.86^{*}$ & 4.95 \\
18 & NK46-44 & 12.89 & 105.30 & -0.07 & 292.00 & $1.06^{* *}$ & 5.26 \\
\hline & $\mathrm{SE}_{\left(g_{i}\right.}$ & 6.32 & - & 9.09 & - & 0.61 & - \\
& $\mathrm{SE}_{\left(g_{i-} g_{j}\right.}$ & 8.94 & - & 12.86 & - & 0.86 & - \\
\hline
\end{tabular}

$* \mathrm{P}=0.05$ and $* * \mathrm{P}=0.01$

Table 5. Specific combining ability (SCA) and mean of the crosses for grain yield, its components and other characters in maize

\begin{tabular}{|c|c|c|c|c|c|c|c|}
\hline \multirow{2}{*}{$\begin{array}{l}\text { Sl. } \\
\text { No. }\end{array}$} & \multirow{2}{*}{ Crosses } & \multicolumn{2}{|c|}{ Days to tassel } & \multicolumn{2}{|c|}{ Days to silk } & \multicolumn{2}{|c|}{ Plant height $(\mathrm{cm})$} \\
\hline & & SCA & mean & SCA & mean & SCA & mean \\
\hline 1. & NK46-2 × BIL28 & -0.89 & 92.50 & -0.49 & 96.00 & 6.37 & 207.30 \\
\hline 2. & NK46-2 × BIL79 & 0.89 & 93.00 & 0.49 & 96.00 & -6.37 & 195.10 \\
\hline 3. & NK46-4 × BIL28 & 0.36 & 97.50 & 0.01 & 100.50 & 3.48 & 191.13 \\
\hline 4. & NK46-4 × BIL79 & -0.36 & 95.50 & -0.01 & 99.50 & -3.48 & 184.70 \\
\hline 5. & NK46-6 × BIL28 & -0.64 & 94.00 & -0.74 & 97.50 & -1.13 & 193.50 \\
\hline 6. & NK46-6 × BIL79 & 0.64 & 94.00 & 0.74 & 98.00 & 1.13 & 196.30 \\
\hline 7. & NK46-7 × BIL28 & -0.39 & 93.00 & -0.24 & 97.50 & -4.78 & 194.10 \\
\hline 8. & NK46-7 × BIL79 & 0.39 & 92.50 & 0.24 & 97.00 & 4.78 & 204.20 \\
\hline 9. & NK46-8 × BIL28 & -0.39 & 92.00 & -0.24 & 96.50 & 0.17 & 197.50 \\
\hline 10. & NK46-8 × BIL79 & 0.39 & 91.50 & 0.24 & 96.00 & -0.17 & 197.70 \\
\hline 11. & NK46-10 × BIL28 & -0.64 & 90.00 & -1.49 & 93.50 & 15.17 & 220.40 \\
\hline 12. & NK46-10 × BIL79 & 0.64 & 90.00 & 1.49 & 95.50 & -15.17 & 190.60 \\
\hline 13. & NK46-12 × BIL28 & 1.11 & 98.50 & 1.26 & 102.00 & -3.38 & 200.20 \\
\hline 14. & NK46-12 × BIL79 & -1.11 & 95.00 & -1.26 & 98.50 & 3.38 & 207.50 \\
\hline 15. & NK46-13 × BIL28 & -1.39 & 97.50 & -0.99 & 99.50 & -10.28 & 194.70 \\
\hline 16. & NK46-13 × BIL79 & 1.39 & 99.00 & 0.99 & 100.50 & 10.28 & 215.80 \\
\hline 17. & NK46-14 × BIL28 & 2.36 & 96.00 & 1.51 & 97.50 & 0.30 & 223.35 \\
\hline 18. & NK46-14 × BIL79 & -2.36 & 90.00 & -1.51 & 93.50 & -0.30 & 223.30 \\
\hline 19. & NK46-18 × BIL28 & -0.39 & 91.00 & 0.01 & 95.50 & -0.73 & 218.00 \\
\hline 20. & NK46-18 × BIL79 & 0.39 & 90.50 & -0.01 & 94.50 & 0.73 & 220.00 \\
\hline
\end{tabular}




\begin{tabular}{|c|c|c|c|c|c|c|c|}
\hline \multirow{2}{*}{$\begin{array}{l}\text { Sl. } \\
\text { No. }\end{array}$} & \multirow{2}{*}{ Crosses } & \multicolumn{2}{|c|}{ Days to tassel } & \multicolumn{2}{|c|}{ Days to silk } & \multicolumn{2}{|c|}{ Plant height $(\mathrm{cm})$} \\
\hline & & SCA & mean & SCA & mean & SCA & mean \\
\hline 21. & NK46-21 × BIL28 & 1.86 & 99.00 & 2.01 & 101.50 & -1.53 & 218.90 \\
\hline 22. & NK46-21 × BIL79 & -1.86 & 94.00 & -2.01 & 96.50 & 1.53 & 222.50 \\
\hline 23. & NK46-22 × BIL28 & 0.11 & 97.50 & 0.01 & 100.50 & -7.18 & 195.30 \\
\hline 24. & NK46-22 × BIL79 & -0.11 & 96.00 & -0.01 & 99.50 & 7.18 & 210.20 \\
\hline 25. & NK46-23 × BIL28 & 0.11 & 93.50 & -0.24 & 96.50 & -11.23 & 190.70 \\
\hline 26. & NK46-23 × BIL79 & -0.11 & 92.00 & 0.24 & 96.00 & 11.23 & 213.70 \\
\hline 27. & NK46-34 × BIL28 & 1.61 & 97.00 & 1.26 & 99.50 & 5.66 & 203.68 \\
\hline 28. & NK46-34 × BIL79 & -1.61 & 92.50 & -1.26 & 96.00 & -5.66 & 192.90 \\
\hline 29. & NK46-39 × BIL28 & -2.64 & 90.00 & -1.24 & 94.50 & 2.86 & 206.80 \\
\hline 30. & NK46-39 × BIL79 & 2.64 & 94.00 & 1.24 & 96.00 & -2.86 & 201.63 \\
\hline 31. & NK46-40 × BIL28 & -1.64 & 88.00 & -0.99 & 92.50 & -0.53 & 211.80 \\
\hline 32. & NK46-40 × BIL79 & 1.64 & 90.00 & 0.99 & 93.50 & 0.53 & 213.40 \\
\hline 33. & NK46-43 × BIL28 & 0.61 & 97.50 & 0.26 & 100.50 & 10.22 & 202.10 \\
\hline 34 & NK46-43 × BIL79 & -0.61 & 95.00 & -0.26 & 99.00 & -10.22 & 182.20 \\
\hline 35 & NK46-44 × BIL28 & 0.86 & 92.50 & 0.26 & 95.50 & -3.48 & 213.40 \\
\hline 36 & NK46-44 × BIL79 & -0.86 & 89.50 & -0.26 & 94.00 & 3.48 & 220.90 \\
\hline & & 1.72 & - & 2.01 & - & 9.57 & - \\
\hline & $\mathrm{SE}_{(S i j-S k l)}$ & 2.43 & - & 2.84 & - & 13.54 & - \\
\hline
\end{tabular}

$* \mathrm{P}=0.05$ and $* * \mathrm{P}=0.01$

Table 5. cont'd.

\begin{tabular}{|c|c|c|c|c|c|c|c|}
\hline \multirow{2}{*}{$\begin{array}{l}\text { Sl. } \\
\text { No. }\end{array}$} & \multirow{2}{*}{ Crosses } & \multicolumn{2}{|c|}{ Ear height $(\mathrm{cm})$} & \multicolumn{2}{|c|}{1000 kernel weight $(\mathrm{g})$} & \multicolumn{2}{|c|}{ Yield (t/ha) } \\
\hline & & SCA & mean & SCA & mean & SCA & mean \\
\hline 1. & NK46-2 × BIL28 & 1.65 & 90.68 & -25.69 & 321.65 & -1.41 & 9.46 \\
\hline 2. & NK46-2 × BIL79 & -1.65 & 86.50 & 25.69 & 355.80 & 1.41 & 12.52 \\
\hline 3. & NK46-4 × BIL28 & -6.03 & 78.40 & 23.89 & 332.50 & $-2.11^{*}$ & 6.37 \\
\hline 4. & NK46-4 × BIL79 & 6.03 & 89.60 & -23.89 & 267.50 & $2.11 *$ & 10.82 \\
\hline 5. & NK46-6 × BIL28 & 0.53 & 98.80 & -7.86 & 304.00 & 1.15 & 7.81 \\
\hline 6. & NK46-6 × BIL79 & -0.53 & 96.88 & 7.86 & 302.50 & -1.15 & 5.74 \\
\hline 7. & NK46-7 × BIL28 & -4.98 & 94.70 & 14.64 & 334.00 & 0.53 & 10.09 \\
\hline 8. & NK46-7 × BIL79 & 4.98 & 103.80 & -14.64 & 287.50 & -0.53 & 9.26 \\
\hline 9. & NK46-8 × BIL28 & -3.08 & 92.30 & $33.90 *$ & 429.00 & 0.77 & 10.46 \\
\hline 10. & NK46-8 × BIL79 & 3.08 & 97.60 & $-33.90 *$ & 343.97 & -0.77 & 9.16 \\
\hline 11. & NK46-10 × BIL28 & 10.42 & 105.30 & 19.00 & 360.23 & $2.13 *$ & 10.99 \\
\hline
\end{tabular}




\begin{tabular}{|c|c|c|c|c|c|c|c|}
\hline \multirow{2}{*}{$\begin{array}{l}\text { Sl. } \\
\text { No. }\end{array}$} & \multirow{2}{*}{ Crosses } & \multicolumn{2}{|c|}{ Ear height $(\mathrm{cm})$} & \multicolumn{2}{|c|}{1000 kernel weight $(\mathrm{g})$} & \multicolumn{2}{|c|}{ Yield (t/ha) } \\
\hline & & SCA & mean & SCA & mean & SCA & mean \\
\hline 12. & NK46-10 × BIL79 & -10.42 & 83.60 & -19.00 & 305.00 & $-2.13^{*}$ & 6.97 \\
\hline 13. & NK46-12 × BIL28 & -2.58 & 96.60 & -24.80 & 315.00 & 0.42 & 9.99 \\
\hline 14. & NK46-12 × BIL79 & 2.58 & 100.90 & 24.80 & 347.36 & -0.42 & 9.39 \\
\hline 15. & NK46-13 × BIL28 & 0.77 & 99.30 & $-39.06 * *$ & 309.10 & -0.66 & 10.47 \\
\hline 16. & NK46-13 × BIL79 & -0.77 & 96.90 & $39.06^{* *}$ & 370.00 & 0.66 & 12.03 \\
\hline 17. & NK46-14 × BIL28 & 1.12 & 114.50 & -17.12 & 273.17 & -0.27 & 7.30 \\
\hline 18. & NK46-14 × BIL79 & -1.12 & 111.40 & 17.12 & 290.19 & 0.27 & 8.08 \\
\hline 19. & NK46-18 × BIL28 & 2.62 & 119.30 & 5.89 & 355.50 & -1.10 & 10.32 \\
\hline 20. & NK46-18 × BIL79 & -2.62 & 113.20 & -5.89 & 326.50 & 1.10 & 12.76 \\
\hline 21. & NK46-21 × BIL28 & 0.57 & 112.60 & 0.14 & 355.00 & 0.70 & 10.71 \\
\hline 22. & NK46-21 × BIL79 & -0.57 & 110.60 & -0.14 & 337.50 & -0.70 & 9.54 \\
\hline 23. & NK46-22 × BIL28 & -3.98 & 90.20 & $-27.61 *$ & 275.00 & -0.15 & 6.96 \\
\hline 24. & NK46-22 × BIL79 & 3.98 & 97.30 & $27.61 *$ & 313.00 & 0.15 & 7.50 \\
\hline 25. & NK46-23 × BIL28 & -0.83 & 98.70 & 16.89 & 378.50 & 0.35 & 8.94 \\
\hline 26. & NK46-23 × BIL79 & 0.83 & 99.50 & -16.89 & 327.50 & -0.35 & 8.49 \\
\hline 27. & NK46-34 × BIL28 & -0.88 & 107.60 & -0.59 & 326.54 & -0.97 & 7.03 \\
\hline 28. & NK46-34 × BIL79 & 0.88 & 108.50 & 0.59 & 310.50 & 0.97 & 9.21 \\
\hline 29. & NK46-39 × BIL28 & 4.17 & 102.70 & 9.56 & 392.85 & 0.14 & 9.34 \\
\hline 30. & NK46-39 × BIL79 & -4.17 & 93.50 & -9.56 & 356.50 & -0.14 & 9.30 \\
\hline 31. & NK46-40 × BIL28 & 1.97 & 108.00 & $-33.93 *$ & 265.00 & 0.53 & 8.30 \\
\hline 32. & NK46-40 × BIL79 & -1.97 & 103.20 & $33.93 *$ & 315.63 & -0.53 & 7.48 \\
\hline 33. & NK46-43 × BIL28 & -0.08 & 98.20 & $37.39^{* *}$ & 381.00 & 0.72 & 11.80 \\
\hline 34 & NK46-43 × BIL79 & 0.08 & 97.50 & $-37.39 * *$ & 289.00 & -0.72 & 10.59 \\
\hline 35 & NK46-44 × BIL28 & -1.33 & 112.70 & 15.38 & 351.98 & -0.76 & 9.51 \\
\hline 36 & NK46-44 × BIL79 & 1.33 & 114.50 & -15.38 & 304.00 & 0.76 & 11.27 \\
\hline & $\mathrm{SE}_{(S i j)}$ & 8.94 & - & 12.86 & - & 0.86 & - \\
\hline & $\mathrm{SE}_{(S i j-S k l)}$ & 12.65 & - & 18.19 & - & 1.21 & - \\
\hline
\end{tabular}

$* \mathrm{P}=0.05$ and $* * \mathrm{P}=0.01$

Considering GCA and SCA effects in preferential direction some lines can be selected for further breeding. NK46-10 was observed with good GCA for days to tasselling and good SCA for yield. NK46-18 was good general combiner for days to tasselling as well as yield. Thus NK46-10 and NK46-18 were selected for earliness and yield. The line NK46-4 was selected for desired GCA effects regarding plant height and ear height and good SCA effect for yield (Table 4 and 
Table 5). On the other hand NK46-2, NK46-13, NK46-43 and NK46-44 were selected for good GCA effects for yield only.

\section{Conclusion}

Seven lines namely, NK46-4 for short plant and ear height and yield, NK46-10 and NK46-18 for earliness as well as yield, NK46-2, NK46-13, NK46-43 and NK46-44 for yield only were selected. These lines may be utilized for advancing to next generation.

\section{References}

Amin, M. N., M. Amiruzzaman, A. Ahmed, and M. R. Ali. 2014a. Evaluation of inbred lines of maize (Zea mays L.) through line $\times$ tester method. Bangladesh J. Agril. Res. 39(4):675-683.

Amin, M. N., M. Amiruzzaman, A. Ahmed, and M. R. Ali. 2014b. Combining ability study in waterlogged tolerant maize (Zea mays L.). Bangladesh J. Agril. Res. 39(2): 283-291.

Amiruzzaman, M. and M. N. Amin. 2011a. Evaluation of inbred lines of field corn through line $\times$ tester method. Annual research report 2010-2011. Plant Breeding Division, BARI, Pp 26-33.

Amiruzzaman, M. and M. N. Amin. 2011b. Evaluation of inbred lines of pop corn through line $\times$ tester method.Annual research report 2010-2011. Plant Breeding Division, BARI, Pp. 34-43.

Das, U. R. and M. H. Islam. 1994. Combining ability and genetic studies for grain yield and its components in maize (Zea mays L.). Bangladesh J. Pl. Breed. Genet. 7(2):41-47.

Hussain, S. A., M. Amiruzzaman and Z. Hossain. 2003. Combining ability estimates in maize. Bangladesh J. Agril. Res. 28(3):435-440.

Ivy, N. A. and M. S. Howlader. 2000. Combining ability in maize. Bangladesh J. Agril. Res. 25: 385-392.

Jahan, N., M. S. Uddin, M. R. Islam, S. Hasna and A. R. M. Saifullah. 2014. Evaluation of inbred line through linextester method. J. Environ. Sci. \& Natural Resources. 7(2): 79-84.

Kempthrone, O. 1957. An Introduction to Genetic Statistics. New York: John Wiley \& Sons, Inc. London: Chapman \& Hall Ltd. Pp. 458-471.

Koppad, S. 2007. Identification of superior parental combinations based on three way cross hybrid performance in maize (Zea mays L.). M.Sc. Thesis. University of Agricultural Sciences, Dharwad. 91p.

Manonmani, S. and A. K. F. Khan. 2003. Studies on combining ability and heterosis in rice. Madras Agric. J. 90(4-6): 228-231.

Mahto, R. N. and D. K. Ganguly. 2001. Heterosis and combining ability studies in maize (Zea mays L.). J. Res. Brirsa Agric. Univ. 13: 197-199.

Narro, L., S. Pandey, J. Crossa, C. D. Leon, and F. Salazar. 2003. Using line $\times$ tester interaction for the formation of yellow maize synthetics tolerance to acid soils. Crop Sci. 43: 1717-1728. 
Parvin, S. 2009. Annual Research Report, Plant Breeding Division, BARI, Pp. 11-18.

Paul, S. K. and R. K. Duara. 1991. Combining ability studies in maize (Zea mays L.). Intl. J. Tropics. Agric. 9(4):250-254.

Rissi, R. D., A. R. Hallauer and R. R. De. 1991. Evaluation of four testers for evaluating maize lines in a hybrid development program. Revista Brasllelia de Genetica. 14(2):467-481.

Roy, N. C., S. U. Ahmed. A. S. Hussain and M. M. Hoque. 1998. Heterosis and combining ability analysis in maize (Zea mays L.). Bangladesh J. Pl. Breed. Genet. 11(1\&2):35-41.

Salgotra, R. K., B. B. Gupta, and P. Singh. 2009. Combining ability studies for yield and yield components in Basmati rice. ORYZA 46(1): 12-16.

Singh, D. N. and I. S. Singh. 1998. Line $\times$ tester analysis in maize (Zea mays L.). J. Res. Agric. Univ. 10: 177-182.

Sofi, P. and A. G. Rather. 2006. Genetic analysis of yield trails in local and CIMMYT inbred line crosses using Line $\times$ tester analysis in maize (Zea mays L.). Asian $J$. Plant Sci. 5(6): 1039-1042.

Suneetha, Y., J. R. Patel and T. Srinivas. 2000. Studies on combining ability for forage characters in maize (Zea mays L.). Crop Res. 9: 226-270.

Shushay, W. A., Z. Z. Habtamu and W.G. Dagne. 2013. Line $\times$ tester analysis of maize inbred lines for grain yield and yield related traits. Asian J. Plant Sci. Res. 3(5):1219.

Talukder, M. Z. A. and B. R. Banik. 2012. Evaluation of inbred lines through line $\times$ tester method. Annual research report 2011-2012. Annual Research Report, Plant Breeding Division, BARI. Pp. 14-21

Talukder, M. Z. A., A. N. M. S. Karim, S. Ahmed, and M. Amiruzzaman. 2016. Combining ability and heterosis on yield and its component traits in maize (Zea mays L.). Bangladesh J. Agril. Res. 41(3):565-577.

Uddin, M. S., F. Khatun, S. Ahmed, M. R. Ali and S. A. Bagum. 2006. Heterosis and Combining Ability in Field Corn (Zea mays L.). Bangladesh J. Bot. 35(2): 109-116.

Wali, M. C., R. M. Kachapur, C. P. Chandrashekhar, V. R. Kulkarni, and S.B. Devaranavadagi. 2010. Gene action and combining ability studies in single cross hybrids of maize (Zea mays L.). Karnataka J. Agric. Sci. 23(4):557-562. 